\title{
Les contours incertains d'un enjeu hégémonique : les ouvriers agricoles dans le Finistère du Front populaire
}

Agricultural workers in Finistère during the Front populaire: The uncertain outlines of a hegemonic challenge

Jean-Paul Sénéchal

\section{CpenEdition}

Journals

\section{Édition électronique}

URL : http://journals.openedition.org/abpo/2499

DOI : 10.4000/abpo.2499

ISBN : 978-2-7535-2236-7

ISSN : 2108-6443

\section{Éditeur}

Presses universitaires de Rennes

\section{Édition imprimée}

Date de publication : 31 décembre 2012

Pagination : 115-136

ISBN : 978-2-7535-2234-3

ISSN : 0399-0826

Référence électronique

Jean-Paul Sénéchal, «Les contours incertains d'un enjeu hégémonique : les ouvriers agricoles dans le Finistère du Front populaire », Annales de Bretagne et des Pays de l'Ouest [En ligne], 119-4 | 2012, mis en ligne le 31 décembre 2014, consulté le 19 avril 2019. URL : http://journals.openedition.org/abpo/2499 ; DOI : 10.4000/abpo.2499 


\title{
Les contours incertains d'un enjeu hégémonique : les ouvriers agricoles dans le Finistère du Front populaire
}

\author{
Jean-Paul SÉNÉCHAL \\ Doctorant en histoire, CRBC-UBO
}

Le Front populaire est synonyme de congés payés, de semaine des 40 heures et d'augmentation des salaires. Cette geste ouvrière et les acquis qui en ont découlé ont porté la focale principalement sur les salariés des centres urbains, au travers de grèves et occupations nombreuses à travers le pays. Dans le Finistère, les grèves des années 1936 ont nourri l'actualité et masqué un phénomène pourtant important : les zones rurales n'ont pas bougé, à quelques rares exceptions près ${ }^{1}$. Pourtant, les salariés y sont nombreux. Parmi eux, une catégorie sociale mérite une attention particulière : les ouvriers agricoles en contact quotidien avec le monde paysan qui les emploie. Dans cette société, véritable bloc rural, qui se vit en permanence dans une situation de confrontation avec le monde urbain, les ouvriers agricoles occupent ainsi une position charnière.

Avant d'aborder les différentes stratégies mises en place, il est indispensable de dresser un tableau de la situation des ouvriers agricoles du département. Première constatation, le Finistère est le huitième département français ayant le plus d'ouvriers agricoles en 1936 avec 63890 personnes ${ }^{2}$. Cette catégorie professionnelle, très hétérogène, comprend le Penn-Ty, ouvrier permanent logeant chez son employeur et cultivant pour ses besoins personnels un minuscule lopin de terre, mais aussi le salarié saisonnier ainsi que le plasennerien de Saint-Pol-de-Léon, véritable forçat de la terre ${ }^{3}$. La répartition géographique de ces ouvriers reflète les besoins en main-d'œuvre : les permanents sont concentrés dans la moitié sud du

1. Quelques grèves éclatent dans les zones rurales, mais ce sont toutes des grèves d'ouvriers chargés de gros chantiers itinérants. Ce ne sont pas des ouvriers ruraux même si quelques-uns d'entre eux sont recrutés localement.

2. Le second groupe professionnel, celui des services publics comprend 35829 personnes. Source : Statistique générale de la France, 1936.

3. Voir ElÉGOËT, Louis, Le Léon, Quimper, Palantine, 2007, p. 157. 
département qui fait également appel à de nombreux saisonniers pour les récoltes de pommes de terre et de petits pois ${ }^{4}$. Dans le nord, la faible taille des exploitations ne nécessite souvent qu'une main-d'œuvre familiale déjà abondante. Cependant, dans quelques rares communes, le nombre des saisonniers dépasse largement la moyenne. C'est le cas à Plougastel-Daoulas, pendant la saison des fraises, et dans la région de Saint-Pol-de-Léon, pour les pommes de terre, les choux-fleurs et les artichauts. La crise affecte particulièrement ce groupe professionnel : 30000 emplois disparaissent entre 1931 et 1936. Les salaires, pourtant déjà très faibles ${ }^{5}$, subissent également les conséquences de la crise.

Malgré leurs conditions d'existence difficiles, les ouvriers agricoles n'attirent pas le regard de leurs contemporains dans les années trente. Cette situation change radicalement dès l'été 1936. Du côté des adversaires du Front populaire, agrariens ou cléricaux, ou chez ses partisans, les initiatives se multiplient, dans une logique de concurrence hégémonique. Pourtant, ce groupe social demeure silencieux alors qu'ailleurs, des ouvriers agricoles se sont fait entendre ${ }^{6}$. La peur de la contagion est donc suffisamment forte parmi les élites catholiques et rurales pour que des mesures préventives soient prises quand elles voient les efforts répétés de la CGT pour pénétrer les campagnes.

\section{Les tentatives d'implantation de la CGT dans le monde rural}

Entre les deux guerres, avant le Front populaire, aucune organisation syndicale d'ouvriers agricoles ne voit le jour. Quant aux grèves, elles sont inimaginables dans la société rurale finistérienne, d'autant que la crise pèse d'un poids très fort. Nul signe de révolte ou de contestation, au moins dans la profondeur des zones agricoles. Cependant quelques éléments, bien rares, témoignent de la transformation des mentalités. Ils ne se manifestent que dans les frontières périurbaines et uniquement dans le sud du département. Là, les propagandistes de gauche peuvent intervenir, comme à Concarneau, où la foire aux gages de 1934 donne lieu à un moment d'agitation qui tient plus du charivari que d'une action concertée ${ }^{7}$. Les ouvriers agricoles côtoient de si près la ville qu'ils en deviennent moins perméables

4. 4200 hectares sont consacrés à la culture des petits pois. La Cornouaille en est le premier producteur du pays ce qui rend nécessaire une main-d'œuvre abondante.

5 . Un valet de ferme reçoit entre 17 et 20 francs pour des horaires qui fluctuent entre 9 et 14-15 heures par jour selon la saison en 1930. Un terrassier quimpérois gagne au même moment 32 francs pour 8 heures de travail.

6. Des grèves ont éclaté en août et septembre 1936 en Île-de-France et dans le Nord. Voir PAXTON Robert O., Le Temps des Chemises vertes : révoltes paysannes et fascisme rural, 1929-1939, Paris, Éditions du Seuil, 1996, 316 p. et FARCY Jean-Claude, " Les grèves agricoles de 1936-1937 dans le Bassin parisien ", dans : La Moisson des autres. Les salariés agricoles aux XIX ${ }^{e}-\mathrm{XX}$ e siècles. Actes du colloque international de Royaumont, Ronald HuBSCHER et JeanClaude FARCY (dir.), Paris, Éditions Créaphis, 1996, p. 303-326.

7. Le Maître, Pierre-Louis, Les Sillons de Beuzec au Pays de Concarneau, Concarneau, Le Maître, 1975, p. 307. 
aux discours distillés par les notables ruraux; la réalité urbaine qu'ils connaissent n'est pas celle d'une société qui méprise les paysans.

Dès que la ville s'éloigne, ces discours sont pris au pied de la lettre. La méfiance va très loin, même quand un emploi est en jeu, alors que le chômage s'étend. À l'exemple de cette tentative avortée de l'office départemental de placement gratuit qui parvient en 1934, alors que le chômage est loin d'avoir disparu, à organiser des départs pour des travaux saisonniers dans le nordest de la France, pour remplacer les ouvriers agricoles traditionnellement employés, des Polonais et des Belges. Seuls 60 ouvriers s'inscrivent sur les listes et la plupart se récusent au moment du départ ${ }^{8}$. Les instituteurs ruraux du département, en tant que syndicalistes, en font eux-mêmes le constat : le paysan a trop souvent été éloigné à leurs yeux de ses véritables intérêts. Et cet état de fait, particulièrement ici dans le Finistère, a pour eux des conséquences graves en terme de rapports de force : une page entière du bulletin du syndicat l'illustre, à partir d'un bilan des élections législatives en juin 1936. Le constat, sous la plume de Pierre L'Haridon, militant très investi dans le travail paysan, est amer : "Nous nous sentons humiliés de voir triompher chez nous l'Église et le Fascisme. Le Finistère n'est-il pas le seul département où un candidat patronné par le cléricalisme militant n'a pu trouver aucun concurrent pour le combattre ${ }^{9}$ ? " Il n'est pas question dans une revue syndicale de faire le bilan des élections à la place des partis politiques, précise le rédacteur de l'article. Cependant, la situation particulière du département a donc alarmé suffisamment le syndicat des instituteurs pour qu'il décide d'accentuer la propagande et d'axer ses priorités vers " les masses rurales qui, trop souvent, ne connaissent que la presse de nos adversaires ». Les instituteurs, "hussards noirs de la République ", ont bien compris l'importance du combat idéologique dans le Finistère et la nécessité d'un travail de fond dans le monde rural. Ils sont très investis dans le travail syndical en direction du monde paysan. Aussi relayent-ils avec enthousiasme l'action de leur syndicat national qui a lancé une souscription pour une campagne antifasciste après les événements du 6 février 1934. Cette campagne débouche sur la création d'un hebdomadaire à destination du monde rural, La Terre Libre. André Delmas, secrétaire général du syndicat, s'en attribue la paternité dans ses mémoires ${ }^{10}$. L'intention est louable, mais les illusions sont certainement très fortes : il est difficile de pénétrer un milieu aussi fermé, quand bien même serait-on un instituteur ${ }^{11}$.

8. Arch. dép. du Finistère, 1N167, Conseil Général, 1935 II, p. 124.

9. Bulletin Mensuel du Syndicat de l'Enseignement Lä̈que du Finistère, juin 1936.

10. Voir Delmas, André, Mémoires d'un instituteur syndicaliste, Paris, Albatros, 1977, p. 242. Delmas, sans le savoir, paraphrase Antonio Gramsci quand il écrit, après avoir rappelé le risque de voir les campagnes basculer dans une voie totalitaire, qu' "en France, à cette époque, aucun régime ne pouvait triompher ou se maintenir sans l'appui de la majorité des ruraux ". Un mémoire de maîtrise a été consacré à cette revue : Sylvie Darroux, "Le Syndicat national des instituteurs à la conquête des campagnes : un exemple "La terre Libre" ", Mémoire de maîtrise, Paris, Université de Paris 1, 1979, 108 p.

11. Jacques Girault démontre combien est illusoire la démarche de ces instituteurs qui portent la bonne parole alors que les voies de socialisation empruntent d'autres chemins, 
La Terre Libre cherche à apporter dans les campagnes une autre voix que celle des élites rurales. La carte des abonnements dans le département dévoile bien la difficulté de la pénétration d'un organe de presse qui remet en cause les hiérarchies acceptées ${ }^{12}$. De nombreuses communes n'ont qu'un ou deux abonnés, sans doute instituteurs. C'est déjà en soi révélateur de l'intérêt des instituteurs pour cette problématique. La revue est effectivement lue au-delà des cercles enseignants, là où les abonnements dépassent les deux ou trois exemplaires (à l'exception vraisemblablement de Brest). Par contre, le Léon reste ainsi à l'écart de leur influence. Deux configurations, en apparence semblables, s'opposent : celle de la région de Saint-Pol-de-Léon-Roscoff et celle de la région de Scaër. Toutes les deux accueillent une très forte population d'ouvriers agricoles et de saisonniers. Les abonnements sont nombreux dans le sud du département, pas dans le nord. La concentration d'ouvriers ne suffit pas à elle seule à créer un climat favorable aux idées sociales portées par la revue des instituteurs. Mais est-ce que le journal parvient, là où il est vendu, à s'attirer des lecteurs chez les paysans? La réponse est oui : la répartition socioprofessionnelle des 75 renouvellements d'abonnements de février 1938 le montre clairement : 27 cultivateurs, 21 instituteurs et 27 artisans, commerçants ${ }^{13}$. Cette intervention dans un milieu extérieur est compliquée, y compris en terme de perspectives d'organisation. La population ciblée n'est visiblement pas définie à l'avance. Paysan, ouvrier agricole, agriculteur sont souvent synonymes pour les syndicalistes enseignants de la CGT. Mais il n'est pas certain que l'objectif soit de structurer la catégorie des ouvriers agricoles. Argumentant pour répondre à des critiques sur la construction de syndicats dans les campagnes, un instituteur de Fouesnant, particulièrement actif sur la question, ne cite que les deux syndicats paysans :

\begin{abstract}
"Des camarades voudraient que nous laissions notre hebdomadaire pour épauler un organe syndical paysan. Ces camarades savent-ils qu'il y a deux syndicats paysans de gauche : la Confédération Nationale Paysanne (C.N.P.) et la Confédération Générale des Paysans Travailleurs (C.G.P.T.)? Quelle organisation aiderions-nous ${ }^{14}$ ?"
\end{abstract}

Est-ce vraiment un malentendu, alors même que l'Union départementale interprofessionnelle CGT s'est lancée de son côté dans un travail de syndicalisation des ouvriers agricoles? Vraisemblablement, la réponse est non. Les instituteurs ne pensent pas aux seuls salariés des champs, mais à ce groupe aux contours incertains de salariés agricoles, de petits propriétaires ou de fermiers, tous dans une situation sociale précaire. Ils partici-

\footnotetext{
sans oublier les contingences économiques qui poussent le paysan ou l'ouvrier agricole vers ceux qu'il pense être les plus utiles à ses intérêts matériels. Voir Jacques Girault, Sur l'implantation du Parti communiste français dans l'entre-deux-guerres, Paris, Éditions Sociales, 1977, p. 43.

12. Voir BARRAL, Pierre, Les Agrariens français de Méline à Pisani, t. 164, Cahiers de la Fondation nationale des sciences politiques, Paris, A. Colin, 1968, 386 p.

13. Bulletin Mensuel du Syndicat de l’Enseignement Lä̈que du Finistère, février 1938.

14. Ibidem.
} 
pent ainsi, à leur corps défendant à perpétuer une vision agrarienne d'une société rurale dans laquelle ils souhaitent pourtant importer un ferment de classe. Cette propagande connaît une fin rapide. La revue, La Terre Libre, connaît des difficultés. Une souscription est lancée au niveau national par le syndicat national des instituteurs. Mais cette campagne pose problème dans le syndicat puisqu'elle est concomitante avec une souscription pour l'Espagne. Certains militants préfèrent privilégier l'action antifasciste de solidarité avec les militants espagnols à la survie d'un journal déficitaire. Le Finistère est l'un des trois seuls départements à avoir atteint l'objectif de ces deux souscriptions. Faut-il y voir un intérêt plus soutenu qu'ailleurs pour maintenir un moyen de propagande en direction des campagnes ${ }^{15}$ ? Ce ne sera pas suffisant. La revue cesse de paraître au printemps 1938, faute de moyens ${ }^{16}$.

Pendant toute l'année 1937, l'Union départementale CGT tente de construire une organisation. Elle profite des foires aux gages ou organise, de manière plus classique, des tournées syndicales ou des campagnes de propagande avec du matériel spécialement adapté au monde agricole. Lors de la foire aux gages de Scaër, le 4 janvier 1937, la CGT organise avec Marquet, le secrétaire de l'Union locale CGT, une réunion après laquelle 200 ouvriers agricoles se sont syndiqués ${ }^{17}$. Elle poursuit l'action engagée les 9 et 10 janvier 1937 et organise une tournée départementale dans une série de communes dont certaines dénotent dans le cadre d'une campagne de développement et de renforcement de l'organisation syndicale ouvrière. La liste est longue, mais tout à fait intéressante : Huelgoat, Carhaix, Pontde-Buis, Châteaulin, Châteauneuf-du-Faou, Quimper, Quimperlé, Rosporden, Douarnenez, Audierne, Pont-L'Abbé, Penmarc'h, Lesconil, Saint-Pol-de-Léon, Morlaix et Landivisiau. Les communes du Léon et celles du Poher n'abritent pas de concentrations ouvrières. Mais le choix est délibéré puisqu'il est prévu " qu'un camarade paysan prendra la parole dans la plupart des meetings ${ }^{18}$ ". C'est une première tentative d'approche systématique. Toute opportunité dans le cadre de cette tentative de pénétration est prise en compte. Un syndicat des meuniers se constitue dans la région brestoise et envoie une délégation au congrès de l'UD qui se tient en début d'année 1937. La direction de l'UD CGT y voit l'opportunité d'établir " un lien sérieux avec nos camarades paysans ${ }^{19}$ ". En août 1937, Charles Berthelot, secrétaire général de l'union départementale CGT, s'empare du sujet, dans un article intitulé " La question paysanne ${ }^{20}$ ", lui donnant ainsi un aspect plus

15. GIRAULT, Jacques, " Le syndicat national des instituteurs et les débuts de la guerre d'Espagne (1936-1937) ", dans La France en mouvement, 1934-1938, sous la dir. de Jean Charles Asselain et Jean Bouvier, Époques, Seyssel, Champ Vallon, 1986, p. 329.

16. Bulletin Mensuel du Syndicat de l'Enseignement Lä̈que du Finistère, avril-mai 1938. Le Finistère a été une des 8 sections départementales à plaider le maintien de La Terre Libre.

17. La Bretagne Ouvrière, Paysanne et Maritime, 30 janvier 1937.

18. Le Finistère syndicaliste, $\mathrm{n}^{\circ} 26$, décembre 1936.

19. Le Finistère syndicaliste, $n^{\circ} 28$, juin 1937.

20. Le Finistère syndicaliste, $n^{\circ} 30$, août 1937. 
officiel et marquant publiquement la volonté de l'Union départementale. À partir de ce moment, l'organisation syndicale s'intéresse de plus près à la question paysanne. Le dimanche 15 août, la commission administrative de la CGT se réunit à Brest en présence de militants plus spécialisés sur la question ${ }^{21}$. Un des militants invités, Coroller, fait un exposé de la situation du Finistère. Les " petits fermiers " constituent la majorité des exploitants agricoles. "Les réactionnaires font accroire à ceux-ci que les ouvriers des villes sont contre les travailleurs des champs. L'effort pour rétablir la vérité s'impose ". Le discours est étonnant. La CGT n'évoque ici que la catégorie des paysans assujettis à un fermage. Par contre, la catégorie des ouvriers agricoles n'est pas non plus citée. Le syndicat interprofessionnel ferait-il comme celui des instituteurs, en privilégiant les agriculteurs propriétaires ou locataires? Se poserait alors un problème de frontières avec le milieu d'intervention du syndicalisme de la $\mathrm{CNP}^{22}$. Il est évident que non, il s'agit ici d'une terminologie inappropriée révélatrice d'une confusion dans l'analyse des couches sociales composant le monde rural. En tout cas, après débat, la commission administrative de la CGT décide d'un plan d'action en plusieurs points. Tous les moyens sont bons pour la propagande ${ }^{23}$. Le Finistère Syndicaliste intègre une rubrique paysanne dès le mois de septembre 1937, et un millier de journaux sont distribués dans les campagnes. La direction du syndicat s'engage donc fortement. L'effort est d'autant plus remarquable que les sollicitations sont nombreuses dans le monde des ouvriers urbains et que les militants syndicaux ont déjà du mal à y répondre. Les conditions historiques ont changé. La CGT voit là une réelle opportunité de se construire en milieu rural et de conquérir idéologiquement une population laborieuse nombreuse.

Charles Berthelot s'implique lui aussi dans cette campagne de mobilisation pour briser l'enfermement des salariés agricoles dans le bloc rural. Il anime une réunion à Plougonven à la fin du mois de septembre 1937, avec Charles Drapier, l'infatigable instituteur animateur des comités de défense laïques, accompagnés d'un orateur paysan : "Camarades paysans, pour dénoncer les menées réactionnaires qui cherchent à dresser les travailleurs des campagnes contre ceux des villes ${ }^{24}$. " Il s'agit bien de

21. Sur les six militants invités à se joindre à la réunion, trois sont nommés dans l'article. Il s'agit de Coroller de Langolen, de Daniel de Saint-Yvi et de Kerbaol de Braspart. Trois autres militants sont également présents. Daniel est instituteur et représentant dans le canton de Rosporden du syndicat des instituteurs. Le Finistère Syndicaliste, 31, septembre 1937.

22. La CNP syndique les fermiers, métayers, ouvriers agricoles, propriétaires travaillant leur propriété eux-mêmes ou avec l'aide d'autrui, jardiniers maraîchers et artisans ruraux qui n'emploient pas plus de deux ouvriers, femmes capables de contracter. Voir BougEARD, Christian, Tanguy Prigent Paysan ministre, Rennes, Presses universitaires de Rennes, 2002, p. 49 .

23. Il est même envisagé d'aller faire des parties de boule dans les villages le dimanche pour essayer de lier contact avec les habitants, à l'image de ce qui se faisait dans le département du Nord avant la guerre. Le Finistère Syndicaliste, n 33, octobre 1937.

24. Annonce parue dans Le Breton Socialiste, 25 septembre 1937. 
chercher à introduire une analyse de classe dans les rapports sociaux du monde rural.

Cependant, cette volonté affichée de s'implanter dans les campagnes se heurte aux désillusions des militants qui se sont emparés de cette mission. Le monde rural reste réellement une terre de mission pour les militants ouvriers. Le ton du rapport moral destiné au congrès de l'UD CGT de 1939, publié en avril 1939 dans Le Finistère Syndicaliste, n'est pas à l'euphorie. Mille exemplaires du journal Le Paysan ont bien été commandés et diffusés pour le $1^{\mathrm{er}}$ mai 1938. Des affiches illustrées, publiées par la Fédération de l'agriculture, ont été envoyées dans les unions locales. Des contacts ont été pris avec la Fédération CGT de l'agriculture pour essayer de construire un syndicat chez les bûcherons et ouvriers forestiers. Cependant, les résultats ne sont pas à la hauteur des espérances. Les militants qui s'étaient engagés à assurer le lien avec le milieu paysan n'ont pas assumé, sur la durée, leur engagement : "Le bureau a constaté, avec regret, que ces relations, cependant agissantes au début, se sont espacées pour disparaître complètement ${ }^{25}$. " La tentative syndicale de pénétration du milieu a fait long feu. Certes, deux syndicats sont créés, ou, du moins leurs statuts sont déposés. Le premier est officiellement déclaré le 6 octobre 1936, c'est le syndicat des travailleurs paysans de Quimperlé. Il s'agit vraisemblablement d'un syndicat affilié à la CGTP ${ }^{26}$. Le second, la section CGT des ouvriers agricoles de Scaër, est constitué le 21 décembre $1936^{27}$.

\section{Les hésitations de la hiérarchie catholique}

Cet échec du mouvement ouvrier laisse-t-il la place aux agrariens de Landerneau? Rien n'est moins sûr, a priori. L'attitude de la hiérarchie catholique est très réservée quant à la direction prise par le syndicat agricole. Le directeur des œuvres, l'abbé Le Goasguen, a un avis très tranché sur le type de syndicalisme nécessaire à la défense des intérêts matériels et moraux du monde paysan :

"On a parlé de neutralité, on a eu peur d'apparaître trop chrétien et de vivre pleinement sa vie. Je dis donc le syndicat des travailleurs des champs aussi bien que le syndicat des travailleurs des villes [...] ne peut être neutre, il doit être chrétien ${ }^{28}$."

Cette position n'est pas partagée par ses pairs des autres diocèses bretons. Le 20 juillet 1936, neuf chanoines, abbés et vicaires venant de cinq diocèses - l'ensemble de la Bretagne historique est là avec les diocèses de

25. Le Finistère Syndicaliste, 49, avril 1939.

26. Le secrétaire déclaré en est Corentin Daeron. Il est cultivateur, ce qui confirme que le syndicat est bien la CGTP, organisation proche du PC et concurrente de la CNP à gauche de l'échiquier syndical agricole. C'est d'autant plus étonnant que Daeron est militant socialiste.

27. Arch. dép. du Finistère, 15 U 25/3.

28. L'Action Catholique du Diocèse de Quimper et du Léon, n 46, juillet 1936. 
Rennes, Vannes, Saint-Brieuc, Nantes et Quimper - se rencontrent pour faire le point sur «l'Organisation agricole dans l'Ouest " dans le cadre d'une journée d'étude. Le chanoine Le Goasguen et l'abbé Favé représentent le diocèse de Quimper ${ }^{29}$. Ce texte offre un éclairage précieux à plusieurs niveaux. Les événements sociaux qui continuent de secouer le pays sont au centre des discussions même s'ils n'y apparaissent qu'en filigrane. La vague de grève n'est pas évoquée, mais le syndicalisme chrétien l'est à plusieurs reprises. Le début de la rencontre est consacré à une description du paysage syndical dans les campagnes. Curieusement, dans le Finistère, l'Office central de Landerneau est présenté comme la seule organisation syndicale ${ }^{30}$. Il n'est fait aucune mention de la Confédération nationale paysanne. Pourtant, la Fédération paysanne du Finistère, qui s'est affiliée à la CNP, s'est constituée en début d'année $1934^{31}$ et s'est régulièrement développée depuis deux ans. Ses actions autour des saisies-ventes lui ont valu plus qu'un succès d'estime. Les hiérarques catholiques considèrent peut-être la CNP comme étrangère au monde du syndicalisme agricole. L'état des lieux fait également mention d'une « Union des Syndicats Paysans de Vendée, rattachée à la C.F.T.C., 40 syndicats, groupant 1700 syndiqués, dont quelques-uns de la Loire Inférieure ». Comme nous le verrons plus loin, la construction d'une structure syndicale spécifique pose de sérieux problèmes aux uns et aux autres. Les différents intervenants n'ont pas tous la même analyse des responsabilités en jeu dans cette situation très instable à leurs yeux. Les élites rurales sont cependant mises en cause au cours de la discussion :

"L'Union centrale des syndicats agricoles [...] ne possède ni hommes, ni argent, ni principes.

Ce manque de principes a provoqué les critiques des vrais syndicalistes chrétiens qui reprochent aux Unions départementales d'avoir absorbé toute leur activité dans des services annexes de coopération, de mutualités, de crédit et d'avoir négligé l'étude et la défense des intérêts professionnels, de l'éducation morale et sociale des syndiqués et l'organisation de la profession. De quoi se plaignent au juste les paysans? Ne traduiraient-ils pas, à leur manière, l'opinion du ministre socialiste : "Parmi vous, il y a trop de mains blanches." "

Les participants cherchent à mesurer l'impact des événements qui fragilisent les positions dominantes dans le monde rural. Ici, se discutent les moyens de prendre en compte, préventivement, certaines aspirations dans les campagnes, à les accompagner pour ne pas voir s'échapper les forces vives du monde rural. La discussion porte sur l'aspect prophylactique face à la menace d'une pénétration des idées sociales de la gauche. Il est donc

29. Un compte rendu dactylographié de 9 pages en est ensuite rédigé, complété " par les notes et observations des Confrères présents ". Arch. Évêché de Quimper, 15Z5, compte rendu dactylographié, sd, 9 pages. Nous avons donc un document relu et donc validé par l'ensemble des participants.

30. L'Office central est annoncé comme regroupant 42000 familles, le Finistère, détenant ainsi la première place en nombre d'adhérents au niveau national.

31. Bougeard, Christian, Tanguy Prigent Paysan ministre, op. cit., p. 49 et suiv. 
nécessaire de faire prendre conscience aux responsables syndicaux de leurs responsabilités. Certains participants à la réunion hésitent cependant à charger les dirigeants des syndicats agricoles, même s'ils ne peuvent que confirmer le constat d'une situation devenue difficile. Afin de ménager les susceptibilités, l'Église se met en cause " sans vouloir jeter la pierre à personne, ni rechercher les responsabilités d'une situation plutôt tendue. Si nous avions à chercher ces responsabilités, on pourrait peut-être les trouver dans le clergé qui, vis-à-vis des organisations agricoles ne semble pas avoir toujours compris le rôle exact qui lui revient ${ }^{32}$ ". À la lecture du document, apparaît en filigrane la question clé, qui ne peut pas se poser comme telle : les propriétaires doivent commencer à répondre aux attentes des deux autres catégories sociales du monde agricole : les fermiers et les ouvriers agricoles. Après avoir pointé du doigt les insuffisances voire les égarements des élites rurales, les participants essayent d'infléchir les politiques à l'œuvre dans les organisations agricoles. Les manques sont patents. La méconnaissance par les dirigeants des syndicats de la doctrine sociale de l'Église explique, pour les cléricaux, ces dysfonctionnements. Y remédier pourrait aider à réguler les tensions sociales déjà perceptibles. Certes, les Unions affiliées à la rue d'Athènes pratiquent une neutralité bienveillante envers l'Église. Cependant, ces mêmes unions sollicitent les prêtres, notamment pour les aider à développer les mutuelles, les caisses agricoles, etc. Cette implication du clergé local engage sa responsabilité aux yeux des populations, sans qu'il y ait de retours satisfaisants pour l'Église. Cela revient à dire que l'Église, dans le monde rural, par l'intermédiaire de ses desservants, sert de caution à une classe sociale contre une autre :

" Il ne s'agit pas d'incriminer la bonne volonté de la plupart des dirigeants, mais connaissent-ils le plus souvent, la doctrine sociale de l'Église? Chrétiens de tradition et de pratiques, ils apportent trop souvent, dans leur action, les préjugés de leur classe dont ils n'ont jamais appris à se défaire, par une étude approfondie des principes sociaux de l'Église ${ }^{33}$. "

La réponse à apporter paraît simple, mais son application plus complexe, avec des réalités de terrain bien différentes selon les diocèses. En Ille-et-Vilaine, un prêtre participe aux réunions les plus importantes d'un point de vue social et se charge de la rédaction du journal. À La Roche-surYon, en Vendée, le prêtre chargé du secrétariat social est systématiquement consulté. Le responsable briochain du syndicat de Landerneau est " en rapports fréquents avec la Direction des œuvres". Ces quelques exemples contrastent avec le tableau, bien plus nuancé, que présente le Finistère où l'osmose est loin d'être réalisée. La question de la syndicalisation concentre les antagonismes. La CFTC, elle-même, hésite sur la position qu'elle doit adopter face à la question complexe de la syndicalisation des " fermiers ou propriétaires cultivant qui sont plutôt des patrons ". L'expérience des syndicats maritimes est évoquée où patrons et salariés se retrouvent dans

32. Arch. Évêché du Finistère, 15Z5, compte rendu dactylographié, sd, 9 pages.

33. Idem. 
la même structure. Quelle issue trouver à la question de l'organisation de groupes sociaux aux intérêts matériels parfois opposés? À l'intérieur de l'Église, les mouvements spécialisés - comme la JAC en Loire-Inférieure qui se rapproche fortement de la CFTC - seraient plutôt portés à créer des syndicats spécifiques. En attendant, le consensus finit par se faire sur une idée avancée par le représentant du diocèse de Rennes : accroître le rôle des commissions paritaires dans les syndicats, afin d'y faire prendre en compte les intérêts de tous. Les signaux reçus des départements sont contradictoires. Un intervenant estime que les dirigeants de l'Union nationale des syndicats agricoles accepteraient d'abandonner les prétentions de l'Union à représenter les ouvriers agricoles. Il note cependant qu'un article récent paru dans Ar Vro Goz laisse penser, au contraire, à un refus total de laisser les mains libres à la CFTC. La doctrine corporatiste s'y oppose en effet. L'intrusion de Dorgères dans le champ syndical amplifie la confusion. "Les Unions semblent beaucoup plus s'occuper de grouper les agriculteurs contre les ouvriers agricoles, sous la direction de Dorgères, que de les aider à s'organiser. " Les participants à la journée d'étude remarquent avec justesse que cette démarche d'exclusion est antinomique d'une approche corporatiste de l'organisation des différentes parties concernées. La question de la CFTC reste donc le point de clivage le plus fort. Pourtant, la menace cégétiste inquiète les responsables religieux bretons. Cependant, les oppositions internes débouchent sur la nécessité de ne rien faire d'autre qu'une étude, tout en minimisant le nombre des ouvriers concernés :

"Une enquête s'impose, car le danger cégétiste pourrait se révéler beaucoup plus proche qu'on ne le croit. Dans le Finistère, Scaër compte plus de 500 ouvriers, Bannalec et les paroisses circonvoisines en comptent au moins autant. Le pays de St Pol compte un très grand nombre de placenners, qui, tous les jours, offrent leur travail sur le marché."

La hiérarchie catholique n'arrive pas à imprimer sa marque sur les choix et orientations dans le monde agricole. Alors même que des consignes strictes de l'Église lui ont été communiquées, Guébriant continue à soutenir Dorgères. Le dirigeant de Landerneau s'était dit " préoccupé de l'attitude d'hostilité prise par certains membres du clergé ${ }^{34}$ " envers le mouvement de Dorgères et toutes ses organisations satellites. Comme le note David Bensoussan, Guébriant s'avance beaucoup en défendant la possibilité pour un jeune jaciste d'être également porteur d'une chemise verte. L'orientation de Dorgères - défense de la famille et du métier, approbation du corporatisme contre un parlementarisme honni - devrait plaider en faveur d'une bienveillance de l'Église. La position de Guébriant, qu'il exprime dans un courrier daté du $1^{\mathrm{er}}$ décembre 1935, est une réponse à l'article paru au mois d'octobre de la même année ${ }^{35}$. La journée d'étude se conclut sur des vœux

34. Bensoussan David, Combats pour une Bretagne catholique et rurale : Les droites bretonnes dans l'entre-deux-guerres, Paris, Fayard, 2006, p. 460.

35. L'Action Catholique du Diocèse de Quimper et du Léon, n 43, octobre 1935, p. 8-9. Cet article, vraisemblablement rédigé par le chanoine Le Goasguen, met les blancs et les 
qui éclairent les compromis acceptables par tous. L'Église doit renforcer sa présence dans les lieux de décision du syndicalisme agricole. La ligue paysanne qui se développe doit être boycottée par les dirigeants et militants de l'Action catholique. Enfin, la construction d'un syndicalisme catholique est souhaitable face aux menaces. C'est le contraire de ce qui s'était dit peu de temps auparavant dans la réunion, preuve des hésitations des dirigeants catholiques qui ne savent plus quelle est la solution : " Il est désirable et, dans certaines régions il est urgent, suivant les directives données par la J.A.C. et l'U.F.C.A. de constituer dans chaque arrondissement des syndicats chrétiens de salariés de l'agriculture. " Une tentative est faite à Saint-Polde-Léon avec la création d'un syndicat des travailleurs de la terre à la fin du premier semestre 1937. Mais celui-ci ne fait pas parler de lui autrement que par un entrefilet dans la presse catholique ${ }^{36}$.

Les cléricaux finistériens, se singularisant des autres clercs bretons dans l'approche du travail syndical, sont en concurrence avec leurs partenaires dans une relation de bloc hégémonique. Entre Landerneau et les cléricaux, les tensions sont permanentes. Landerneau ne souhaite pas s'opposer de front à la hiérarchie catholique, mais ne veut pas plier et reste fermement attaché à son autonomie d'action. L'organisation agrarienne oppose à l'Église plutôt une inertie totale qui produit les mêmes effets dans la pratique : l'impossibilité de construire un syndicalisme chrétien en milieu rural dans le Finistère. Les tergiversations de la hiérarchie catholique finistérienne, qui n'est pas confrontée à la même situation sur le terrain que les autres diocèses, ne sont pas nouvelles. De nombreux échanges ont eu lieu sur la nécessité du développement et sur le rôle de la JAC au début des années trente ${ }^{37}$. Rien n'est donc réglé. Le chanoine Le Goasguen se heurte visiblement ici à un obstacle dans le déploiement de l'action catholique. La tentative de l'Église de contrôler Landerneau ne peut se faire de manière directe. Le poids de l'Office central, la personnalité de son dirigeant, ses convictions profondes sont autant d'écueils qui ne peuvent être franchis. Cela n'empêche pas Le Goasguen de rester campé sur ses positions. Il n'hésite pas à l'écrire à Inizan, le député de la 3 e circonscription de Brest, dans une correspondance privée, au début de l'année 1938. Le directeur des œuvres, qui travaille sous le contrôle direct de M $^{\text {gr }}$ Duparc, n'a pas

rouges dans le même panier. L'article est rédigé de manière à ne pas être lu comme une condamnation unilatérale du dorgérisme. Après avoir critiqué vertement les communistes et socialistes, l'article donne la parole à une femme, qui, elle, s'en prend au Parti agraire.

36. Le Petit Breton, 4 juillet 1937.

37. Voir Berger, Suzanne, Les Paysans contre la politique, Paris, Éditions du Seuil, 1975, p. 122-134. Suzanne Berger cite une lettre de l'aumônier de la JAC pour les Côtes-du-Nord qui révèle parfaitement la différence d'appréciation avec Le Goasguen et Favé du coté finistérien : "Ce qui fait, je crois, que nos appréciations sur l'Office central ne sont pas tout à fait les mêmes, c'est que vous désirez, vous, qu'il fût ouvertement catholique, tandis que nous nous contenterions (jusqu'à ce que l'autorité ecclésiastique nous ait donné tort) qu'il le fût en fait, sans l'afficher, qu'il s'inspirât, dans tous les cas où la morale est intéressée, de la morale chrétienne, sans plus. ", op. cit., p. 131. 
« confiance dans l'Office central, préoccupé des problèmes techniques, bien plus que de l'adaptation de l'apostolat dans un milieu donné 38 ". Dans le cercle des élites rurales et cléricales, ces divergences sont vraisemblablement connues. Elles restent cependant internes aux réseaux dirigeants.

\section{L'Office central de Landerneau s'adapte pour ne rien changer}

Pendant la période du Front populaire, Landerneau s'applique à anticiper toute possibilité d'installer la lutte des classes dans les campagnes. L'Office central s'intéresse particulièrement à suivre l'évolution des conditions de fermage, mais garde également à l'œil le monde des ouvriers agricoles.

\section{La une de l'hebdomadaire Ar Vo Goz sur la réunion de Pleyben (janvier 1938)}

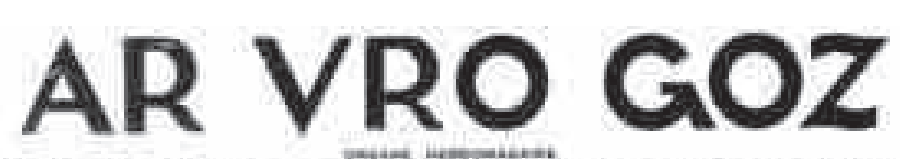

OE LYNION DES SYNBICATS AGAICOLES DU FINISTAREET DES COTES-DUNORO

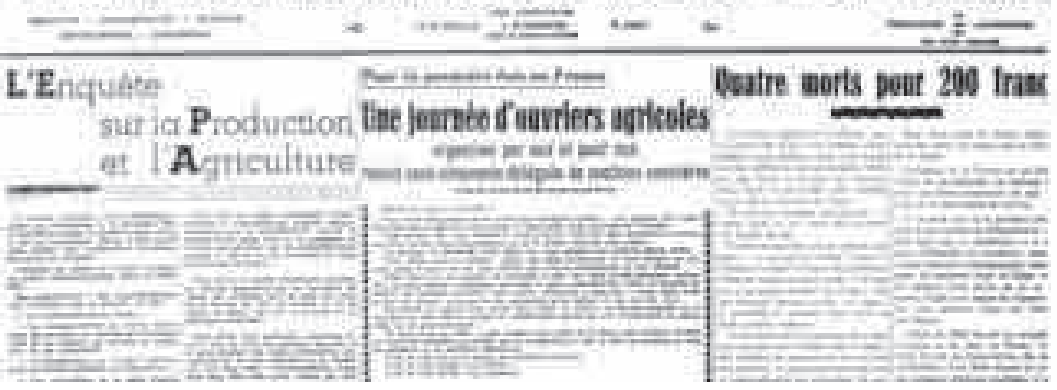

Pour prévenir les tentatives d'implantation de sections syndicales d'ouvriers agricoles importées par la société urbaine, le monde rural met en place ses propres structures, sous son contrôle strict. Les dirigeants de l'Union des syndicats agricoles du Finistère ont très vite compris les risques de pénétration des idées socialistes dans les campagnes. Dans le pays, les campagnes s'agitent et des grèves éclatent. Dès le mois de juillet 1936, le journal de l'Office central fait un compte rendu des grèves qui éclatent dans les campagnes, s'attachant à démontrer qu'elles sont importées des villes, mouvement "fomenté toujours par des non-paysans ". Le journal dresse un tableau apocalyptique des conséquences des grèves à coup d'imageschocs, comme pour la grève de la Somme :

38. Arch. Évêché de Quimper, 22K2, lettre de Le Goasguen à Inizan, député du Finistère, 19 février 1938. 
"Hier il était dangereux de circuler, les travailleurs étaient terrorisés; chaque soir, autour de feux de joie, hommes et femmes dansaient la "Carmagnole"; le drapeau rouge était hissé sur les fermes... On se serait cru à Moscou ${ }^{39}$."

Les dirigeants de Landerneau cherchent à déconsidérer les grévistes, mais, en même temps, ils mesurent la portée de ces événements. Ce ne sont pas des jacqueries cette fois-ci, mais bien l'irruption du prolétariat et donc de la lutte des classes dans les campagnes. Deux premières réponses apparaissent très vite : rappeler tout le temps que les intérêts des paysans sont les mêmes, quelle que soit leur position sociale et aussi organiser des structures de contrôle syndical des ouvriers agricoles, en leur ouvrant les syndicats déjà existants, pour ne laisser aucune place à toute autre organisation.

Mais, en même temps, il est nécessaire pour les agrariens de prendre quelques mesures afin de montrer que l'initiative de l'amélioration du niveau de vie dans le monde agricole provient des élites rurales soucieuses de garantir à tous des conditions d'existence acceptables. À certaines périodes, il est nécessaire de passer des compromis; Guébriant a parfaitement compris cet impératif. En cette période de déséquilibres, le compromis social doit être renégocié et il vaut mieux prendre l'initiative pour ne rien lâcher d'essentiel. "Sans doute, l'hégémonie présuppose-t-elle qu'on tienne compte des intérêts et des tendances des groupes sur lesquels s'exercera l'hégémonie, que l'on parvienne à un certain équilibre de compromis, en d'autres termes que le groupe dirigeant fasse des sacrifices d'ordre économico-corporatif, mais il est également hors de doute que ces sacrifices et ce compromis ne peuvent pas concerner l'essentiel ${ }^{40}$. " Il faut donc agir sur tous les fronts : la mise en place, par Landerneau, de commissions paritaires dans le département répond au vote par les députés de l'extension des conventions collectives à l'agriculture. Cette loi menace de rompre le dialogue bien inégal entre employeurs et ouvriers agricoles. Elle accorde au ministre de l'Agriculture " un pouvoir dictatorial ${ }^{41}$ ". Ces commissions abordent également la question cruciale des salaires. La menace est trop sérieuse. Plutôt que de s'affronter au syndicalisme ouvrier, l'Office central de Landerneau accompagne le mouvement, preuve d'un sens politique affûté. François-Marie Jacq, qui en est l'ancien secrétaire général, reconnaît lors d'un entretien que ces structures sont mises en place " pour couper l'herbe sous le pied de la CGT avant qu'elle ne mette ses griffes sur les ouvriers ${ }^{42}$ ". Même la CFTC est à craindre dans cette stratégie de contrôle total. À la fin de l'année 1936, les ouvriers travaillant chez les exportateurs de Saint-Pol-de-Léon s'engagent avec l'Union dépar-

39. Ar Vro Goz, 14 et 28 juillet 1936.

40. Antonio Gramsci, Cahiers de prison, cahiers 10, 11, 12 et 13, Paris, Gallimard, 1978, p. 388 .

41. Ar Vro Goz, 7 mars 1937.

42. Entretien accordé par François-Marie Jacq à Suzanne Berger le 24 janvier 1964. BERGER Suzanne, Les Paysans contre la politique, op. cit., p. 102. 
tementale des syndicats CFTC dans une négociation pour obtenir une augmentation de salaire et une convention collective. 500 ouvriers menacent de faire grève ${ }^{43}$. C'est du jamais vu dans cette cité, cœur du Léon et fief des Guébriant. La grève n'a pas lieu et les salariés reprennent le travail aux conditions accordées par les employeurs, mais l'alerte a été chaude. Un tel mouvement n'aurait pas été imaginable s'il n'y avait eu cette atmosphère de revendications liée aux grèves qui perdurent depuis juin 1936. C'est le signe que, peut-être, une époque se termine. Mais les élites rurales ont dû percevoir également deux autres éléments derrière ce conflit. D'une part, l'UD CFTC n'aurait jamais pris la responsabilité de prendre part à ce conflit sans l'accord explicite de l'Évêché. D'autre part, si la grève avait commencé, elle aurait entraîné des conséquences non seulement symboliques sur le pouvoir réel des élites rurales, mais également économiques, en bloquant les circuits de commercialisation des productions agricoles.

Les structures syndicales spécifiques sont rapidement créées par le syndicat corporatiste. À la fin de l'année 1936, 75 sections regroupent 567 domestiques et ouvriers agricoles ${ }^{44}$. La pression sociale est forte. Un exemple illustre bien les processus de domination à l'œuvre. Dans la commune de Pleuven, le maire, Jean Chalony, préside aux destinées de la section locale du syndicat. Il est en même temps responsable des Comités de défense paysanne. Lors d'une réunion, au début du mois de décembre 1936, il aborde la nécessité de syndiquer et d'organiser les ouvriers agricoles. Au cours de la même réunion est discutée la question des allocations familiales. Chaque patron cultivateur doit verser une somme proportionnelle aux ouvriers de plus de 16 ans qu'il emploie. Il la verse à une caisse de compensation de son choix. Cette caisse répartit les allocations ensuite. Bien sûr, Landerneau a mis en place une caisse : la Caisse régionale de Bretagne d'allocations familiales mutuelles agricoles ${ }^{45}$. Comment, dans ces conditions, faire preuve de la moindre indépendance? À la mi-juillet 1937, le président de l'Union des syndicats agricoles annonce au chanoine Le Goasguen que les résultats ont dépassé les espérances puisque les sections ouvrières comptent 2400 adhérents. Ils ont été multipliés par quatre en six mois $^{46}$. En 1939, dans un courrier, l'Union des syndicats explique en détail au préfet du Finistère la structuration et le fonctionnement de ces sections ouvrières et annonce un chiffre de syndiqués en recul :

"Quand les ouvriers syndiqués sont au nombre de cinq, ils se constituent en section, à l'intérieur du syndicat, section qui a son président et son secrétaire et qui s'occupe spécialement des questions intéressant les salaires.

43. Arch. dép. du Finistère, 10M69, dossier expéditeurs de Saint-Pol-de-Léon et lettre de l'union départementale CFTC, 15 janvier 1937.

44. Arch. dép. du Finistère, 7M68, Compte-rendu de la commission paritaire, 19 novembre 1936.

45. Ar Vro Goz, 14 et 13 décembre 1936.

46. Arch. Évêché de Quimper, 6K5, lettre de Guébriant au chanoine Le Goasguen, 29 juillet 1937. 
Dans le Finistère, nous comptons environ 103 sections ouvrières et 2119 ouvriers syndiqués. Une grande partie de ces derniers est constituée par ce qu'on appelle les "Penn-Ty" c'est-à-dire les ouvriers mariés qui logent avec leurs familles dans une petite maison appartenant au propriétaire ${ }^{47}$."

Les chiffres que l'organisation de Landerneau annonce pourraient paraître satisfaisants. Mais par rapport au nombre de salariés concernés, l'implantation ne correspond pas à la position hégémonique de Landerneau. Comparés à ceux de la CGT, qui n'a pas les mêmes moyens au niveau national, les résultats laissent apparaître des résistances. En effet, le recensement de 1936 comptabilise 63868 ouvriers et employés de l'agriculture. Le taux de syndicalisation serait donc 3,32\%, en utilisant les chiffres annoncés par l'Union des syndicats agricoles en 1939. Cependant, la CGT, sur l'ensemble du territoire, arrive à un chiffre de 2,55\%. Plus faible, certes, mais, dans le premier cas, le taux syndicalisation de l'Union des syndicats agricoles porte sur son territoire alors que pour la CGT, le taux est calculé sur l'ensemble du pays, zones d'influences et déserts syndicaux mêlés ${ }^{48}$. Ce relatif succès démontre-t-il une résistance passive à Landerneau? La prise d'une carte à la CGT serait pour un Penn-Ty le plus sûr moyen de perdre en même temps son travail et son logement. Faute de pouvoir choisir son syndicat, l'ouvrier agricole, dans sa grande majorité, ne prend pas sa carte au syndicat de Guébriant où il se retrouverait avec son patron, la distinction entre section ouvrière et section exploitant étant factice. La mise en place de structures syndicales a été rapide. Le tempo n'est plus le même quand il s'agit d'appliquer les nouvelles lois sociales. Congés payés, salaires, quarante heures, contrats collectifs appliqués à l'agriculture servent à l'Office central pour dresser les agriculteurs contre le monde urbain. Mais Landerneau doit quand même s'emparer de ces sujets pour ne pas laisser l'État les gérer. David Bensoussan arrive au même constat à propos de cette pratique à vocation hégémonique : "La volonté d'organiser de manière exclusive l'encadrement de la paysannerie oblige les dirigeants de l'Office central à s'emparer de toute avancée sociale afin d'en contrôler l'application et d'éviter l'ingérence de l'État ou de groupes rivaux ${ }^{49}$. " Pour les congés payés, l'Office déploie de longs argumentaires techniques qui complexifient le problème rendant indispensable le statu quo : " Il semble bien qu'il soit de l'intérêt de l'employeur comme de l'employé que rien ne soit changé. Si le domestique s'absentait pendant un certain laps de temps, le patron de nos petites exploitations devrait faire seul le travail.

47. Arch. dép., 7M78, lettre de l'Union des syndicats agricoles du Finistère au préfet, 17 juin 1939.

48. Le chiffre a été calculé par Prost Antoine, La C.G.T. à l'époque du Front populaire, Cahiers de la fondation nationale des sciences politiques, Paris, 1964, p. 67.

49. David Bensoussan, « Le syndicalisme agricole entre conservatisme et progressisme : le projet syndical du comte de Guébriant dans la première moitié du $\mathrm{Xx}^{\mathrm{e}}$ siècle ", dans : PITOu, Frédérique (dir.), Élites et notables de l'Ouest XVI'-XX siècle, Entre conservatisme et modernité, Rennes, PUR, 2003, p. 198. 
De plus, quel serait l'emploi du temps des domestiques pendant quinze jours consécutifs ${ }^{50}$ ?"

Ar Vro Goz commence une véritable campagne de communication envers les ouvriers agricoles avec son numéro du 4 août 1936. Dans un article, "Les actuels devoirs du syndicalisme agricole", trois devoirs sont énumérés. Le premier est " de se préoccuper avec une extrême attention du sort des ouvriers agricoles ", dans des commissions paritaires mixtes parce que "l'intérêt économique commun favorise les rapprochements, d'autant plus que les catégories chevauchent les unes sur les autres " et que ces commissions empêchent "l'intrusion d'étrangers à la profession ". Le second, le développement du syndicat corporatiste répond à la volonté hégémonique de la CGT qui " entraînée par le communisme, joue une grosse partie dans les campagnes. Elle sait parfaitement que si elle n'arrive pas à entraîner la paysannerie, c'est celle-ci qui, finalement, aura le dernier mot ". Le dernier est un devoir de défense paysanne qui implique de fait la possibilité à un passage à la violence si les dirigeants de Landerneau l'estiment nécessaire. À la suite de cette déclaration qui recherche le consentement tout en évoquant la coercition, sept longs articles sont consacrés pendant l'automne et le début de l'hiver 1936 aux domestiques de ferme ${ }^{51}$. En effet, un glissement sémantique se fait jour : les ouvriers agricoles se font appeler domestiques par les dirigeants du syndicat. L'explication est intéressante quand elle est confrontée à cette série d'articles tout à fait inhabituelle dans le journal agricole. La pression des événements fait nécessité, mais le rédacteur ne peut s'empêcher de signifier à l'ouvrier qu'il appartient à la maison : "Ce mot "domestique" [...] vient du latin "domus" (maison) pour désigner ceux que d'autres appellent tout simplement les ouvriers [...] Il en est même qui font corps, pour ainsi dire, avec la maison, avec la ferme; ils y demeurent quand le maître change ${ }^{52}$." Ces articles préparent le terrain en justifiant le syndicat unique puis expliquent longuement le projet des agrariens de Landerneau en matière d'instances de discussions et de négociations : les commissions paritaires. L'Office central crée la commission paritaire très tôt, concurrente, de fait, des commissions de conciliation mises en place par le gouvernement. Cette commission paritaire permet notamment d'éviter la négociation de conventions collectives. Une première réunion se tient le 29 octobre 1936 en présence de dix employeurs et de dix ouvriers venus de tout le département.

"C'est sans doute la première fois, en France, qu'employeurs et employés se réunissent, non pas en deux blocs opposés pour confronter leurs revendications, mais en une commission unique pour discuter froidement des intérêts réciproques de chacune des parties. Nous avons le droit d'en être fiers $^{53}$."

50. Ar Vro Goz, 4 août 1936.

51. Ar Vro Goz, 18 et 25 octobre, 8, 13 et 20 novembre, 13 et 20 décembre 1936.

52. Ar Vro Goz, 18 octobre 1936.

53. Ar Vro Goz, 8 novembre 1936, Cité par Hélène Hirrien, Un syndicat pour les ouvriers agricoles : du syndicat des Ouvriers agricoles d'Élliant à la FGA de la CFDT (1936-1990), 
Guébriant préside la première réunion. Les différents points d'actualité y sont traités. Le principe d'un contrat type de travail préparé par la commission est accepté, mais il ne définit que les lignes principales. Les parties au contrat devront s'entendre sur les points de détail, ce qui laisse une très grande latitude à l'employeur toujours en position de force dans ce type de négociation. Enfin, comble d'indépendance, la commission qui comprend 10 ouvriers, dans une lettre adressée au ministre de l'Agriculture, " approuve le principe de 15 jours de congés payés annuels; mais s'élève, à l'unanimité, contre l'obligation de 7 jours au moins consécutifs ". Une seconde réunion de la commission paritaire se tient le 19 novembre sur les salaires et la durée du travail ${ }^{54}$. Les salaires sont calculés sur une base qui tient compte pour moitié de l'indice du coût de la vie et l'autre moitié de l'augmentation moyenne des produits agricoles. Là encore, les négociations sont laissées à l'initiative des commissions locales. Quant à la durée du travail, la commission départementale la fixe selon les mois de l'année et les exigences du métier. Bien entendu, ces horaires ne sont pas obligatoirement exécutoires et doivent être pris comme des conseils. Dès l'année 1937, l'Office central se fait plus discret sur le sujet. Quelques réunions sont encore organisées, notamment à Pleyben, en janvier 1938. 150 délégués des sections ouvrières sont réunis pour l'occasion dans la commune que Landerneau présente comme la première de France, avec 125 adhérents. Cette réunion est mise en relief par Landerneau.

Cependant, cette organisation corporatiste des relations sociales n'a pas été simplement une réaction de rejet du Front populaire ou une tentative pour étouffer toute velléité naissante de mise en place d'organisations syndicales autonomes. Ce réflexe - il faut bien parler de réflexe puisque la mise en place est quasi immédiate : quelques semaines après le début des grèves ouvrières dans le département - s'explique également par une vision de la société et des rapports sociaux guidée par "le remplacement de l'État libéral par un régime corporatif ${ }^{55}$ ". Ainsi Landerneau met au point un bail type pour résoudre un conflit ancien entre fermiers et propriétaires; attitude identique à la mise en place de la commission paritaire qui s'installe à l'automne 1936. Malgré toutes les précautions, Guébriant craint de devoir faire face à une fuite des ouvriers. Un argument revient souvent : "Le privilège intolérable dont bénéficient les employés de l'État sous forme d'allocations très élevées aux familles nombreuses, par la semaine des

op. cit., p. 28. Hélène Hirrien détaille la mise en place de cette commission paritaire.

54. Ar Vro Goz, 20 novembre 1936.

55. "Landerneau n'avait jamais nettement séparé la vie privée et les activités susceptibles d'une réglementation autoritaire. À ses yeux, aucun aspect de la vie rurale n'échappait de plein droit au contrôle professionnel. Toutefois, tant que la paix sociale n'était pas troublée, l'Office central laissait, dans la pratique, de vastes zones de la vie paysanne hors du contrôle de l'organisation professionnelle, s'en remettant aux structures traditionnelles pour assurer l'harmonie entre les classes rurales. Lorsque les divergences s'approfondirent et que le contenu de la paix sociale commença à être entamé, l'Office central se préoccupa des moyens de faire respecter ses décisions. " BERGER Suzanne, Les Paysans contre la politique, op. cit., p. 167-169. 
quarante heures, les congés, etc. " vide les campagnes ${ }^{56}$. Dans la période, les agrariens ne se limitent pas à intervenir dans le champ des rapports sociaux négociés. Ils maintiennent également la pression, se servant des Comités de défense paysanne pour bien signifier que leur contrôle sur la société rurale est complet. La violence dans les campagnes finistériennes est réelle en 1936. Les chemises vertes de Dorgères n'arrêtent pas d'organiser des réunions de propagande ou des actions auxquelles répondent les forces de gauche. Elles se vantent de regrouper dans le Finistère de nombreux jeunes paysans prêts à en découdre. Mais la Défense paysanne organise-t-elle uniquement les propriétaires ou plus largement l'ensemble de ceux qui travaillent la terre? L'organisation factieuse s'enorgueillit de rassembler toutes les couches sociales et cite en exemple la commune de Trégunc, " en majorité communiste, [où] il y a aujourd'hui 47 chemises vertes parmi lesquelles de nombreux ouvriers agricoles ${ }^{57}$ ". La mention des ouvriers n'est pas anodine : les dorgéristes y attachent visiblement de l'importance, sans doute de manière symbolique, en montrant qu'ils arrivent à recruter en terre rouge même parmi les ouvriers agricoles, qui devraient pourtant être plus enclins à se rapprocher du Parti communiste, comme le sous-entend l'article du journal agrarien. Cette situation que l'Office central laisse se développer et même encourage, exposerait tout ouvrier agricole qui chercherait à défendre ses intérêts par la grève. Ar Vro Goz publie ainsi le compte rendu d'une réunion de 200 dirigeants agricoles représentant 50 syndicats de la région de Tréguier, théâtre de mouvements de grève parmi les ouvriers chargés du teillage de lin. Dans son intervention, Guébriant est très clair : " À la force, s'il est besoin, il faut répondre par la force ${ }^{58}$. " L'Office central n'a pas hésité à citer en exemple, en juillet 1936, les Comités de défense paysanne de Dorgères " qui ont volé au secours de leurs frères menacés " par les grèves ${ }^{59}$. Les ouvriers agricoles savent ce qui les attend s'ils montrent la moindre velléité de mobilisation. Ainsi, sous la coupe de Landerneau, s'établit un subtil équilibre entre consentement et coercition dans les campagnes. Dans le Nord-Finistère, cela passe plutôt par le consentement alors que dans le sud et dans le Trégor, comme l'ont montré Christian Bougeard dans son ouvrage sur Tanguy-Prigent et Robert Paxton sur les chemises vertes, la force des chemises vertes est un de leurs arguments politiques. D'ailleurs, la hiérarchie catholique n'est pas dupe. Malgré de très claires mises en garde, l’Office central continue son

56. Ar Vro Goz, 27 décembre 1936. La lettre du Président de la Chambre d'agriculture du Finistère, qui n'est autre que Guébriant est publiée avec un titre évocateur : "L'exode vers l'arsenal".

57. Le Progrès agricole de l'Ouest, 5 janvier 1936. Robert O. Paxton relève, quant à lui, que le mouvement dorgéristes comporte très peu d'ouvriers. Dorgères prétendait au contraire que les Chemises vertes étaient composées essentiellement d'ouvriers alors qu'il s'agissait la plupart du temps de fils de paysans travaillant à la maison. PAXTON Robert O., Le Temps des chemises vertes : révoltes paysannes et fascisme rural, 1929-1939, op. cit., p. 199.

58. Ar Vro Goz, 7 mars 1937.

59. Ar Vro Goz, 28 juillet 1936. 
jeu trouble avec les troupes de Dorgères. Cette proximité ne peut échapper aux ouvriers agricoles.

" Il est assez étrange que le département où les Chemises Vertes ont pris le plus, c'est justement celui où il y a le plus de syndiqués. Nous savons pertinemment que les dirigeants des sections locales de ces syndicats ont été appelés eux-mêmes à préparer les voies à Dorgères et à encourager la jeunesse à s'enrôler dans les Chemises Vertes.

Même, après les avis formels de la Direction des Euvres, le Président de l'Office Central de Landerneau ne perd pas une occasion de chanter la louange des Chemises Vertes."

Enfin Guébriant joue sur un autre paramètre pour assurer une paix sociale dans les campagnes, ou du moins dans celles où son organisation est en position de monopole. Il s'assure que l'offre de main-d'œuvre soit suffisante et même légèrement excédentaire, mais jamais déficitaire. Par deux fois des déséquilibres menacent et des mesures sont prises à chaque fois pour assurer un équilibre. À la fin de la guerre, la crise des fermes - trop de paysans reviennent du champ de bataille alors que les terres disponibles se sont raréfiées - entraîne Guébriant à chercher une solution pour limiter la pression démographique. La crise prévisible risque d'ébranler l'équilibre. Alors le dirigeant agricole mobilise les réseaux et chacun, député, évêché et syndicaliste agrarien, qui plus est noble, joue son rôle pour chercher à résoudre le problème qui s'annonce avec l'éviction des fermiers de leurs terres. Se forme là un véritable bloc agraire qui cherche, par le consentement, à trouver une solution pour résoudre une équation complexe : des hommes reviennent de la guerre alors que les terres qu'ils ont travaillées leur sont enlevées. La solution est d'autant plus urgente que des mouvements de protestations ont éclaté dans les Côtes-du-Nord pour les mêmes motifs. Une perspective s'ouvre avec de nombreux avantages : l'émigration. Vincent Inizan intervient à ce sujet à l'Assemblée nationale le $1^{\text {er }}$ février 1921, souhaitant que soit évité le départ hors de France, mais aussi " le chemin des villes où il y a déjà pléthore et où sévit avec assez d'intensité la crise du logement et du chômage. Ils aiment la terre, ils veulent lui rester fidèles. Gardons-les dans notre pays pour notre terre à nous ${ }^{60}$ ". De son côté, Guébriant écrit à l'évêché le 10 février 1921 qu'il est nécessaire de se préoccuper des conditions sociales de l'accueil des migrants ${ }^{61}$. Il demande à l'Église de se concerter pour préparer la venue des paysans et l'encadrer. Et l'opération est un succès. L'émigration vers l'Aquitaine permet de réduire la pression démographique et le chômage et de voir se produire dans le Léon les incidents qui éclatent dans les Côtes-

60. LE BAIL, Sylvain, Cours de Breizh, aux bretons d'ici et d'ailleurs, Collection en partage, Ploërmel, Les oiseaux en papier, 2009, p. 161.

61. Un premier convoi part vers le Périgord en juin 1921. Fait révélateur, ils sont accompagnés par trois " pilotes "; ils n'ont aucune autonomie. Une fois sur place trois groupes sont constitués pour être fermiers dans trois secteurs différents du département. Les léonards d'un coté, les cornouaillais, bigoudens et quimperlois de l'autre et enfin ceux de Châteaulin et Châteauneuf-du-Faou, ibidem, p. 174. 
du-Nord au même moment pour les mêmes motifs. Plus de 500 familles quittent ainsi le Léon ${ }^{62}$. La logique à l'œuvre est la même lorsqu'en 1937 la CFTC de Saint-Pol-de-Léon organise le départ de petits cultivateurs, d'ouvriers agricoles et d'ouvriers emballeurs, réduits à l'inactivité par la fin de la saison des pommes de terre et des artichauts. Elle récidive en 1938 et plus de 700 ouvriers s'engagent dans la campagne sucrière dans le Loiret, l'Eure et Loire et dans l'Yonne ${ }^{63}$. À l'inverse, avec la mobilisation, les bras commencent à manquer dans les campagnes. C'est l'opportunité pour certains ouvriers d'essayer de faire jouer en leur faveur la loi de l'offre et de la demande. En avril 1940, Guébriant écrit au préfet pour lui rappeler qu'ils ont eu un entretien au sujet d'ouvriers agricoles, profitant d'une maind'œuvre rare, qui exigent jusqu'à 18000 ou 20000 francs par an, nourris et logés. "Nous avons examiné ensemble ce qui pourrait se faire pour mettre bon ordre à cette situation ${ }^{64}$. " Il joint à son courrier copie d'un ordre de réquisition du préfet de Seine-et-Oise, en espérant qu'il puisse inspirer au préfet du Finistère un acte similaire qui ruinerait toute velléité de négociation des ouvriers agricoles. Peine perdue, le préfet lui répond rapidement qu'il envisage bien de prendre un arrêté rendant officielle l'entraide à la culture. S'agissant des salaires, " dont l'exagération ne fait aucun doute ", il ne voit aucune autre solution que de les faire fixer par le comité départemental de la production agricole, mais il ajoute que c'est une arme à double tranchant puisque les salaires en vigueur dans le Finistère sont très variables et " accusent d'une région à une autre, des différences parfois très sensibles ${ }^{65}$ ". Dans une situation de pénurie de bras, les difficultés de l'élite rurale à maintenir son ascendant deviennent réelles.

Les ouvriers agricoles ne se sont pas mobilisés pendant le Front populaire. Plusieurs explications doivent être avancées; il serait illusoire de chercher une cause unique à cette totale absence de front social. Les conditions économiques dans les campagnes ne sont pas étrangères à cette attitude : les revenus des ouvriers agricoles du département ont progressé, même modestement, pendant le Front populaire. De plus, vivant à la campagne, ils souffrent moins de la hausse du coût de la vie qui réduit considérablement dans les villes les bénéfices des hausses de salaire. Cette amélioration du niveau de vie a été consentie par crainte de mouvements sociaux dans les campagnes d'autant plus facilement que le niveau de vie de l'ensemble des paysans a évolué positivement.

Au-delà de ces premiers éléments de réponse, comment expliquer cette discrétion sociale des ouvriers agricoles? Guy Bajoit, reprenant la thèse de Albert Hirschman, avec son triptyque d'attitudes : exit, voice, ou loyalty, l'enrichit d'une quatrième, l'apathy pour ceux et celles qui ne peuvent faire

62. ELÉGOËT, Louis, Le Léon, op. cit., p. 169.

63. [http://www.roscoff-quotidien.eu/Roskodatesg.htm].

64. Arch. dép. du Finistère, Lettre de Guébriant, 27 avril 1940.

65. Arch. dép. du Finistère, projet de lettre du préfet, sd. 
défection, ni protester tout en étant loyaux par contrainte de situation ${ }^{66}$. Même en ne se situant pas dans une logique utilitariste, cette apathie s'observe bien sur le terrain : les tentatives d'implantation syndicale de la CGT, comme celles de la hiérarchie catholique ont fait long feu. Seul le syndicat de Guébriant réussit à construire une structure syndicale, expression d'une attitude empreinte de loyalty. Mais l'explication ne peut se résumer à ce type d'attitude. Béatrice Hibou, travaillant sur la domination, a démontré qu'un comportement, a priori à l'inverse de ses intérêts propres, pouvait être motivé par une conjonction de facteurs ou de " complications " de la domination $^{67}$. Les raisons en sont multiples, complexes. Le désir de sécurité, l'instrumentalisation de la crainte de l'autre, c'est-à-dire de la société urbaine, sont des ressorts sur lesquels jouent les appareils hégémoniques. Les pratiques culturelles sont au cœur du processus qui permet d'y parvenir. L'économie politique de la domination joue également un rôle important pour ce groupe subalterne dont une des caractéristiques est la plasticité : les ouvriers agricoles se nourrissent aussi de l'espoir de sortir de leur condition. Cette porosité entre les groupes sociaux permet l'ascension sociale et réduit d'autant les velléités d'émancipation. Mais, en période de crise sociale, les groupes hégémoniques savent que leur légitimité est " fluctuante et largement circonstancielle, sensible au temps ${ }^{68}$ " et que les concessions possibles sont de fait réduites. D'où cette attention soudaine portée à des salariés auparavant invisibles, dès l'irruption des grèves ouvrières. Le dyptique consentement-coercition se fait plus apparent alors. La force, ici celle des Chemises vertes, n'est pas que symbolique. La crise révèle également des stratégies divergentes à l'intérieur du bloc rural.

Dans le sud, quelques traces de prise de distance avec les élites rurales commencent à transparaître. La création éphémère de syndicats spécifiques en est l'expression. Cependant, les résultats électoraux de communes rurales, comme Scaër ou Élliant, avec une très forte population d'ouvriers agricoles parmi les électeurs, en atténuent largement la portée. Ces communes offrent en effet des scores importants aux agrariens en 1936, avec $46,20 \%$ pour la première et $42,47 \%$ pour la seconde. Le milieu n'est donc pas totalement hermétique aux tentatives d'approche des organisations ouvrières, mais cela reste très relatif.

Les ouvriers agricoles sont bien les forgotten men $^{69}$ des études rurales pour reprendre une expression utilisée lors du premier colloque qui leur a été consacré, mais également des forgotten women dans une activité où les

66. BAJoIT, Guy, « Exit, voice, loyalty... and apathy. Les réactions individuelles au mécontentement ", Revue française de sociologie, 29-2, 1988, p. 325-345.

67. Hiвou, Béatrice, Anatomie politique de la domination, Paris, Édition La Découverte, 2011, 298 p.

68. HiBou, Béatrice, Anatomie politique de la domination, op. cit., p. 31.

69. Expression utilisée dans l'introduction au colloque « Les salariés agricoles aux 19e $20^{\mathrm{e}}$ siècles " des 13 et 14 novembre 1992. Voir HuBSCHER Ronald et FARCY Jean-Claude (dir.), La Moisson des autres. Les salariés agricoles aux XIX'-XX siècles. Actes du colloque international de Royaumont, op. cit. 
emplois féminins représentent près de la moitié des effectifs ${ }^{70}$. Ils en arrivent à s'oublier eux-mêmes. La roue de l'histoire tourne cependant. Les ouvriers agricoles s'organisent en masse après la Seconde Guerre mondiale : un syndicat CGT est créé dans le département. Il rassemble 5000 des 20000 ouvriers du Finistère selon l'ancien secrétaire de l'Union départementale, Fanch Tanguy ${ }^{71}$. Ce syndicat réussit à conclure un accord avec les représentants départementaux de la CGA (Confédération générale de l'agriculture) qui fixe une limite annuelle du travail à 2400 heures et établit un règlement qui précise notamment le repos hebdomadaire, les congés et les salaires ${ }^{72}$.

\begin{abstract}
RÉSUMÉ
Pendant l'entre-deux-guerres, les ouvriers agricoles représentent une partie importante mais au combien silencieuse du salariat finistérien. Ce groupe professionnel n'apparaît cependant que de manière périphérique pendant le Front populaire. Il est pourtant l'objet de nombreuses attentions du mouvement ouvrier, de la hiérarchie catholique comme des agrariens. La CGT tente vainement de construire une organisation de salariés dans les campagnes. La hiérarchie catholique s'en inquiète mais tergiverse entre la construction d'une CFTC ou le soutien aux sections d'ouvriers agricoles mises en place par Landerneau. La rapidité de réaction du syndicat agricole clôt les débats. L'organisation de Guébriant a, encore une fois, anticipé les difficultés avec une réponse s'inscrivant parfaitement dans son optique agrarienne. Nous avons ici un groupe social, les ouvriers agricoles, qui n'a pas conscience de sa force collective potentielle. La lutte pour obtenir son consentement participe de la lutte pour l'hégémonie, à l'échelle du département, en s'adaptant au réalités locales.
\end{abstract}

\title{
ABSTRACT
}

In the inter-war years, agricultural workers represented an important but silent part of the wage earners in Finistère. However this occupational group appeared only peripherally during the Popular Front. Yet it was the subject of much attention from the labor movement, the Catholic hierarchy and the agrarians. The trade union, CGT, vainly tried to build an organization of workers in the countryside. The Catholic hierarchy was worried but it equivocated between building a trade union, CFTC, and supporting sections of agricultural laborers implemented by the town of Landerneau. The responsiveness of the Union des syndicats agricoles closed the debate. The organization of Hervé Budes de Guébriant had again anticipated difficulties with an answer that fitted perfectly its agrarian perspective. Here we had a social group, agricultural workers, that was unaware of its potential collective strength. The fight for obtaining its consent participated in the struggle for hegemony, at the level of the département, by adapting to local realities.

70. 23907 femmes sont recensées en 1936 comme employées ou ouvrières en agriculture, contre 39961 hommes. Le groupe " travail des étoffes, vêtements ", qui vient juste après en taille avec 3828 salariées, est six fois moins important.

71. TANGUY, Fanch, La CGT dans le Finistère, La bataille commence, t. 1, Union départementale CGT du Finistère, 1986, p. 81.

72. Ibidem, p. 111-112. 